\title{
Preface
}

In recent years, Massive Open Online Courses (MOOC) are appearing as a new way of learning, more dynamic and more accessible. These new courses are characterized with a great diversity of topics and a huge number of enrolments.

This book discusses and addresses the challenges in the MOOC design, implementation, management and deployment. Additionally, the book explores the impact of new methodologies and frameworks used in the life cycle of a MOOC, ranging from well-designed synchronous virtual classrooms to assessment, gamification and collaborative services and tools.

This comprehensive and timely publication aims to be an essential reference source, building on the available literature in the field of E-learning and MOOCs management while providing for further research opportunities in this dynamic field. It is hoped that this text will provide the resources necessary for practitioners, educators, teachers, technology developers and managers to adopt and implement dynamic and flexible MOOCs, using integrated learning environments capable of giving timely feedback and adjusting learning based on the students' learning profile and pace.

\section{THE CHALLENGES}

MOOCs are an emergent topic and we can read plenty of success stories. However, this new paradigm raises serious questions regarding the assessment and the large dropout rates. This book focuses on different aspects of the MOOC life-cycle process and how the assessment and retention issues were addressed. Apart from these well-known issues, other problems are identified and discussed as well. This new paradigm raises serious questions regarding the monitoring of student progress and its timely feedback. In fact, studies show that novice students in an e-learning system might feel being isolated from the teachers and other students, because of the lack of dynamic interactions components in the system design. This issue increases when we talk about complex domains (e.g. computer programming domain). These types of issues lead to a negative impact on the students' outcome. 


\section{DESCRIPTION AND ORGANIZATION OF THE BOOK}

This book presents a comprehensive and recent view of the emerging trends, techniques, and tools for Massive Open Online Course (MOOC) to foster the learners progress and knowledge in specific and complex domains. At the same time, it identifies new trends on this topic from pedagogical strategies to technological approaches. The book is organized in ten chapters. A brief description of each of the chapters follows:

Chapter 1 discusses elements of the landmarks of MOOCs and OERs highlighting concepts and definitions to the innovation purposes in higher education. The chapter reflects on the wider problematizations on OER and MOOC in higher education aiming to five dimensions: spatio-time-content; theoretical models; principles of pedagogical innovation; economic aspects and fundamentals of collaborative culture.

Chapter 2 proposes a lifecycle for the successful development and implementation of MOOCs. The lifecycle covers both the MOOC development process as well as its management, considering both pedagogy and software engineering. It covers essentially three stages: development, management and improvement. The development phase involves the design and testing of the MOOC. The management phase involves the implementation and deployment of the MOOC. Finally, the improvement phase, includes the preservation, evaluation and optimization of MOOC.

Chapter 3 shares a set of guidelines to assist designers, content producers and researchers in developing intuitive, accessible, contextual and effective multimedia content. This aggregation of guidelines will help the previous agents to design solutions faster and in a more efficient and manner. Equally as important as the documentation of guidelines is the generation of new ones, since culture is constantly changing and the guidelines must adapt to those changes.

Chapter 4 surveys the use of gamification in e-learning as a powerful tool for user's engagement. The chapter enumerates a set of game elements and mechanics, that can be mapped from games to other domains such as medicine, marketing and, specially, education. Beyond the extensive list of game components, a list of successful gamified e-learning applications is presented to sustain the usefulness of this approach to foster learner's retention and motivation. A closer and interesting look at the future of gamification in the education context is also presented.

Chapter 5 presents a deep research on the design of teaching/learning methods using gamification. In fact, authors advise consider gamification techniques to motivate and encourage students, as well as Learning Styles to improve their learning performance. The chapter also depicts on the incongruences and incompatibilities that are triggered when applying both Gamification and Learning Styles while designing a MOOC. 
Chapter 6 enumerates several approaches for the decreasing of dropout rates in MOOC environments. These approaches are based on differentiated instruction that considers individual learners' needs in order to ensure a more suitable environment for each learner. Based on these approaches, the design process is initiated by examining and analyzing learners' characteristics, and defining a set of learning paths within MOOC. After deployment, the system can provide pedagogical support to learners to sustain their learning.

Chapter 7 presents a study to evaluate the motivation of Arabic MOOC learners in a language online course. The findings of the study reported a set of motivating factors for students to enroll in a MOOC for languages learning, such as, the desire to improve skills, to learn a new topic, to get a better job, to prepare for a better future and curiosity. Another result from the experiment included in the chapter was that investigating the type of learners' motivation helps teachers and institutions to stimulate them and maintain motivation levels throughout the course.

Chapter 8 surveys the state of the art related to technology-based approaches used to teach and learn foreign languages. In this context, MOOCs are increasingly being used since, but there is a huge demand for the use of more sophisticated environments that will allow users, for instance, to support ubiquitous mobile social interaction. These new ways of communication and interaction will increase the quality learning process not only in the field of languages, but also in other domains.

Chapter 9 presents the M100S (Math Without Stress), an e-learning project from the Polytechnic of Porto. The chapter describes the full development process of the project organized by several phases. For each phase, the authors share all the steps to create or refine the composed education resources. In the end, the set of open educational resources were integrated in a MOOC platform to help learners in the Math subject. Nowadays, the course is still online and a set of statistical data was shared and analyzed showing interesting and promising results. The guidelines presented in the chapter could help others to create dynamic educational resources for domains where technology is crucial to overcome the intrinsic difficulties typically associated to complex domains.

Chapter 10 explores the selection and the integration of a plagiarism detection software in learning management systems. For this task, the authors used a constructive group decision method called nominal group technique. This work could be a useful checklist for other higher education institutions that rely on open source software for the management of their academic and administrative affairs. 


\section{CONCLUSION}

The proposed book aims to share new approaches and methodologies for the design and management of MOOCs. At the same time, it identifies new trends on this topic from pedagogical strategies to technological approaches. The proposed book could be used as a valuable resource for practitioners and as a reference for research scholars, teachers and students that rely on technology to teach/learn specific subjects.

\section{Ricardo Queirós}

Polytechnic Institute of Porto, Portugal 